\title{
Impact of Bundle Structure on Performance of on-Chip CNT Interconnects
}

\author{
Nisha Kuruvilla ${ }^{1}$ and J. P. Raina ${ }^{2}$ \\ ${ }^{1}$ Department of Electronics Engineering, College of Engineering, Chengannur, Kerala 686121, India \\ ${ }^{2}$ Center for Nanotechnology Research, VIT University, Vellore, Tamil Nadu 632 014, India \\ Correspondence should be addressed to Nisha Kuruvilla; nisha@ceconline.edu
}

Received 11 January 2014; Revised 16 April 2014; Accepted 4 May 2014; Published 5 June 2014

Academic Editor: Jorge M. Seminario

Copyright (C) 2014 N. Kuruvilla and J. P. Raina. This is an open access article distributed under the Creative Commons Attribution License, which permits unrestricted use, distribution, and reproduction in any medium, provided the original work is properly cited.

\begin{abstract}
CNTs are proposed as a promising candidate against copper in deep submicron IC interconnects. Still this technology is in its infancy. Most available literatures on performance predictions of CNT interconnects, have focused only on interconnect geometries using segregated CNTs. Yet during the manufacturing phase, CNTs are obtained usually as a mixture of single-walled and multi-walled CNTs (SWCNTs and MWCNTs). Especially in case of SWCNTs; it is usually available as a mixture of both Semi conducting CNTs and metallic CNTs. This paper attempts to answer whether segregation is inevitable before using them to construct interconnects. This paper attempt to compare the performance variations of bundled CNT interconnects, where bundles are made of segregated CNTs versus mixed CNTs, for future technology nodes using electrical model based analysis. Also a proportionate mixing of different CNTs has been introduced so as to yield a set of criteria to aid the industry in selection of an appropriate bundle structure for use in a specific application with optimum performance. It was found that even the worst case performance of geometries using a mixture of SWCNTs and MWCNTs was better than copper. These results also reveal that, for extracting optimum performance vide cost matrix, the focus should be more on diameter controlled synthesis than on segregation.
\end{abstract}

\section{Introduction}

Though many in the semiconductor industry have predicted the failure of Moore's Law during the past decade, several IC manufacturers today are in the process of commercializing sub-45 nm IC technologies [1]. Development work for the $32 \mathrm{~nm}$ and $22 \mathrm{~nm}$ device nodes is well underway. Yet successful IC development beyond these feature sizes is by no means guaranteed because of the fundamental limits imposed by the laws of physics. As the cross section of conventional electrical wires reduces, its performance getting limited due to physical phenomena's including electronic scattering, surface scattering from boundaries of ultra-narrow conductors. These factors inhibit their electronic conduction and act as serious roadblocks to Moore's Law extension at the most fundamental level. The resistivity of copper interconnects, with cross-sectional dimensions of the order of mean free path of electrons ( $40 \mathrm{~nm}$ in copper at room temperature) in current technologies, increases rapidly under the combined effects of enhanced grain boundary scattering, surface scattering, and the presence of highly resistive diffusion barrier layers. This mounting interconnect delay has always been a major bottleneck in designing interconnects meant for transmitting signals with frequencies of the order of terahertz. The associated reliability issues have become the driving force behind the search for alternative interconnect materials [1]. Although the field of interconnect nanotechnology for terahertz speeds is still in its infancy, the potential emerging technologies explored so far are 3D interconnects, optical interconnects, and interconnects using nanowires and tubes. These carbon nanotubes, with their many attractive properties [2-4], have emerged among the frontrunners. Preliminary theoretical, simulation, and experimental studies are required to explore the potential of these materials as futuristic IC interconnects.

As a result of the fabrication process a realistic CNT bundle is a mixture of single-walled/multiwalled CNTs. The fabrication results demonstrated in $[5,6]$ indicate that a realistic nanotube bundle consists of a mixture of SWCNTs 


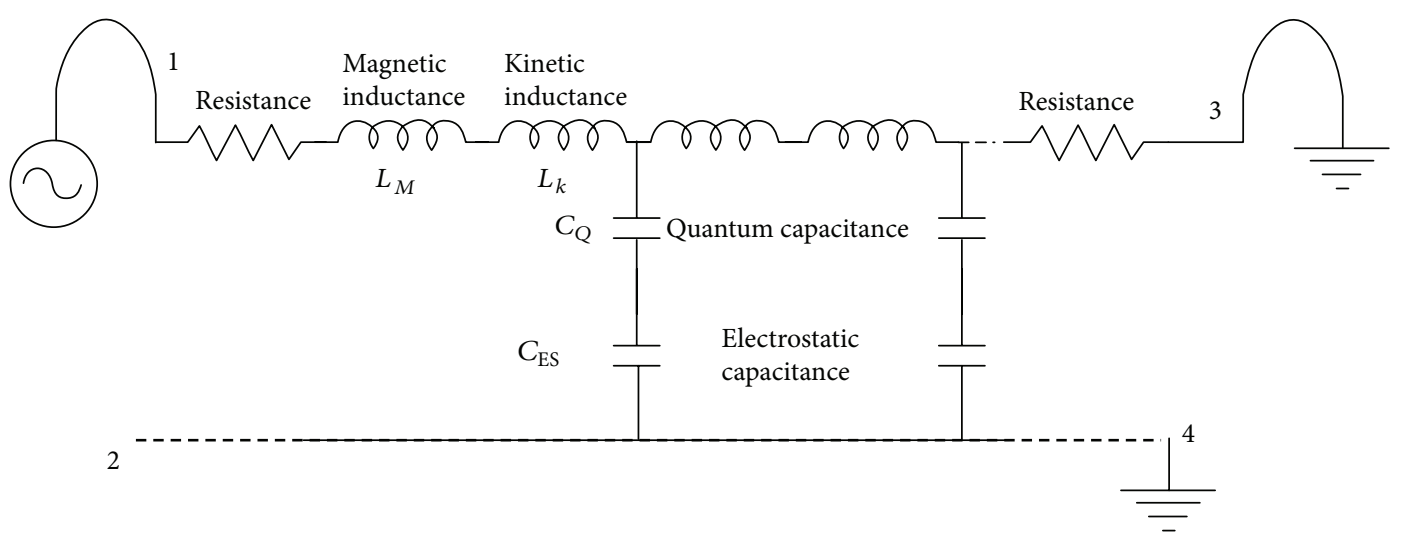

FIgURE 1: Equivalent circuit model for a CNT interconnect [10].

(semiconducting and metallic) and MWCNTs and these also indicate a possibility of diameter controlled synthesis of CNTs. Hence it is necessary to include both kinds of CNTs in a performance analysis of CNT bundles to provide realistic estimates for the industry. The existing literature on modeling and performance analysis of CNT interconnects has mostly focused on interconnects made of single SWCNT or bundles of segregated SWCNTs or bundles of SWCNTS with identical diameters [2-4] and to a lesser extent on bundles of MWCNTs [7]. Though models for parasitic effect of mixed CNT bundles have been formulated in the relative variations in performance between interconnects using segregated SWCNTs, mixed CNTs have not been studied $[8,9]$. These studies dealt with segregated SWCNTs or were based on the assumption that CNTs in a bundle are mixed randomly.

This paper evaluates the performance variation of different geometries of CNT bundled interconnects using phenomenological electrical models, under the constraints of process variations. Performance analysis of various geometries of bundled CNT interconnects are carried out in this work. This work includes six different CNT interconnect geometries includes, bundles made of, segregated and unsegregated SWCNTs, segregated and un-segregated MWCNTs, mixed CNTs of random nature and also bundles formed by mixing of SWCNTs and MWCTS in known proportions. It also evaluates the performance of mixed bundles formed of SWCNTs of various diameters and bundles of MWCNTs of various diameter ratios. The performance variation associated with these six possible geometries of CNT interconnects has not been compared in any available literature till date. The comparison between segregated and unsegregated bundles helps in correlating fabrication cost and performance reliability of these interconnects geometries.

This paper is organized as follows. Section 2 discusses the diameter dependent electrical model used for the evaluation of performance of CNT interconnects of various geometries. Section 3 deals with the problem and the methodology of solutions. It also discusses the mathematical models used for the calculation of various parameters for simulation and worst case analysis of mixed bundles constructed under proportionate mixing of SWCNTs and MWCNTs. Section 4 presents the results obtained, and Section 5 discusses the conclusion.

\section{Circuit Model for Mixed CNT Bundle}

Equivalent single conductor circuit model of CNT interconnect used for performance evaluation is given in Figure 1.

The details of the calculation of various parasitic elements are discussed below. Studies proved that usually one-third of the shells has metallic nature and the rest are semiconducting. The shells of CNTs in a bundle have random chiralities and each of which has a number of conducting channels depending on the diameter and metallic tube ratio. Each conducting channel exhibits either intrinsic conductance $\left(G_{Q}\right)$ and ohmic conductance $\left(G_{o}\right)$ or scattering conductance which is dependent on mean free path $\lambda$ and tube length $(l)$, where $G_{o}=\left(2 q^{2} / h\right)(\lambda / l)$ and $G_{Q}=2 q^{2} / h \cong 1 / 12.9 \mathrm{~K} \Omega(h$ is the planks constant and $q$ is the charge of an electron and mean free path $\lambda$ which is diameter dependent).

The conductance offered by a conducting shell is [9]

$$
G_{\text {shell }}(d, l)= \begin{cases}2 G_{Q} & l \leq \lambda \\ 2 G_{o} & l>\lambda\end{cases}
$$

The total conductance of all the shells is [9]

$$
\begin{aligned}
G_{\text {MW }}\left(D_{\text {outer }}, l\right) & =\sum_{N_{\text {shell }}} G_{\text {shell }}\left(d_{i}, l\right) \\
& =\sum_{d_{i}=D_{\text {inner }}}^{D_{\text {outer }}} G_{\text {shell }}\left(d_{i}, l\right),
\end{aligned}
$$

where the numbers of shells $\left(N_{\text {shell }}\right)$ are determined by inner tube diameter $D_{\text {inner }}$ to outer tube diameter $D_{\text {outer }}$.

The total conductance offered by a mixed bundle is

$$
G_{\text {bundle }}=\int G_{\mathrm{MW}}\left(D_{\text {Outer }}, l\right) N\left(D_{\text {Outer }}\right) \partial D_{\text {Outer }}
$$

where the tube count in a bundle $N\left(D_{\text {Outer }}\right)$ is [9]

$$
N\left(D_{\text {outer }}\right)=\frac{N_{\text {bundle }}}{\sigma D_{\text {outer } \sqrt{2 \pi}}} \exp \left[-\frac{1}{2}\left(\frac{D_{\text {outer }}-\overline{D_{\text {outer }}}}{\sigma D_{\text {outer }}}\right)^{2}\right]
$$


(a Gaussian distribution with a mean $\overline{D_{\text {outer }}}$ and a standard deviation of $\sigma D_{\text {outer }}$ ) [8, 9]. Parasitic conductance effect due to intershell tunneling is not considered in this analysis.

The inductance offered by a CNT has two components: magnetic and kinetic. The magnetic inductance is due to the presence of magnetic field inside and between the tubes. Kinetic inductance is due to the kinetic energy of electrons and is given by [11]

$$
\begin{aligned}
& L_{k, \text { Bundle }} \\
& =\frac{L_{k, \text { chan }}}{\sum_{\text {All } D_{\text {outer's }}}\left(N\left(D_{\text {outer }}\right) * \sum_{N_{\text {shell }}} N_{\text {chan } / \text { shell }}\right)}(\mathrm{nH} / \mu \mathrm{m}) .
\end{aligned}
$$

The magnetic inductance $L_{m \text {,Bundle }}$ is estimated from the partial self-inductance of each tube and mutual inductance among the tubes. The total inductance of the bundle is the sum of the bundle kinetic and magnetic inductance:

$$
L_{\text {total }}=L_{k, \text { Bundle }}+L_{m, \text { Bundle }} \text {. }
$$

The capacitance of a nanotube bundle consists of both a quantum capacitance $\left(C_{q}\right)$ and an electrical capacitance $C_{E}=$ $2 *\left(C_{g}+C_{C}\right)$. The quantum capacitance is equivalent to the quantum electrostatic energy stored in the nanotube when it carries current. The quantum capacitance of each conducting channel is $C_{q 0}=e^{2} / h v_{F} \approx 50 \mathrm{aF} / \mu \mathrm{m}$. The total quantum capacitance of the bundle is

$$
C_{q}=C_{q 0} * N_{\text {channel } / \text { bundle }} * l_{b},
$$

where $l_{b}$ is the length of bundle. The electrical capacitance between a nanotube bundle and adjacent nanotube bundles depends on the bundle geometry and spacing between bundles. This has been evaluated using CNIA tool based on an empirical formula as given below:

$$
\begin{array}{rl}
C_{g}=\epsilon_{\mathrm{ox}} & * l_{b} \\
\times & {\left[\left[\frac{w_{b}}{h_{b}}+2.04 *\left(\frac{s_{b}}{s_{b}+0.54 * h_{b}}\right)\right]^{1.77}\right.} \\
& \left.*\left(\frac{t_{b}}{\left(t_{b}+4.53 * h_{b}\right)}\right)^{0.07}\right] \\
C_{c}=\epsilon_{\mathrm{ox}} & * l_{b} \\
\times & {\left[1.14 *\left(\frac{t_{b}}{s_{b}}\right) * e^{\left(-4 * s_{b} /\left(s_{b}+8.01 * h_{b}\right)\right)}+2.37\right.} \\
& *\left(\frac{w_{b}}{\left(w_{b}+0.31 * s_{b}\right)}\right)^{0.28} *\left(\frac{h_{b}}{\left(h_{b}+8.96 * s_{b}\right)}\right) \\
& \left.* \exp \left(-\frac{2 s_{b}}{\left(s_{b}+6 * h_{b}\right)}\right)\right],
\end{array}
$$

where $\epsilon_{o x}=$ permittivity of the oxide, $l_{b}=$ length of the bundle, $w_{b}=t_{b}=$ width of the bundle, $h_{b}=$ height of the bundle above the ground plane, and $s_{b}=$ spacing between bundles. Consider

$$
C_{E}=2 *\left(C_{g}+C_{C}\right)
$$

The total capacitance of the bundle is

$$
C_{\text {Bundle }}=\frac{C_{q} * C_{E}}{C_{q}+C_{E}} .
$$

All these parasitic effects bundled CNT interconnects which are assumed to be densely packed were evaluated for various interconnect geometries using carbon nanotube interconnect analyzer (CNIA) tool [11].

\section{Problem Definition and Calculation of Various Parameters}

CNTs are obtained as a mixture of single-walled and multiwalled CNTs (SWCNTs and MWCNTs) during its synthesis process [6]. However industry currently focuses only on interconnects consisting of segregated SWCNTS or MWCNTS [12-14]. This paper attempts to check whether segregation of CNTs is really essential before using them as interconnects. If segregation can be eliminated, then this will save both total production costs and time.

Initially the performance of fully and partially metallic bundles consisting of segregated SWCNTs, unsegregated SWCNTs of random diameters, segregated MWCNTs, and randomly mixed SWCNTs and MWCNTs as per ITRS predicted dimensions for the years up to 2022 was evaluated by varying the metallic tube ratio. In case of bundles of MWCNTs and mixed ones, the variation in inner to outer diameter ratio $\left(D_{\min } / D_{\max }\right)$ was also considered. The average diameter of the tubes are kept constant as $1.5 \mathrm{~nm}$ and a realistic nanotube density of $10^{12}$ tubes $/ \mathrm{cm}^{2}$ in the bundle [8]. Comparing these results, it was found that unsegregated randomly mixed bundles outperformed geometries. At this stage, the effect of mixing in ratios was not clear. In order to reveal the effect of mixing on performance, further studies were carried out using proportionately mixed CNTs. The optimum mixing ratio in terms of comparative performance was evaluated by analyzing the performance of the bundles made of known proportion of SWCNTs and MWCNTs. A pictorial view of various CNT interconnect geometries used for this analysis is given in Figure 2. This work will help the industry in selection of an appropriate bundle structure for its use in specific application. These results were also compared with the performance of copper interconnects.

The size of the interconnect geometry was as per the ITRS prediction given in Table 1 . The entire study was carried out at room temperature with carbon to carbon spacing of $1.42 \mathrm{~A}$ and shell spacing of $3.4 \mathrm{~A}$, under tight binding energy of $3 \mathrm{ev}$.

\section{Results}

Initially performance analysis is to check out performance variation between fully and partially metallic bundles con- 


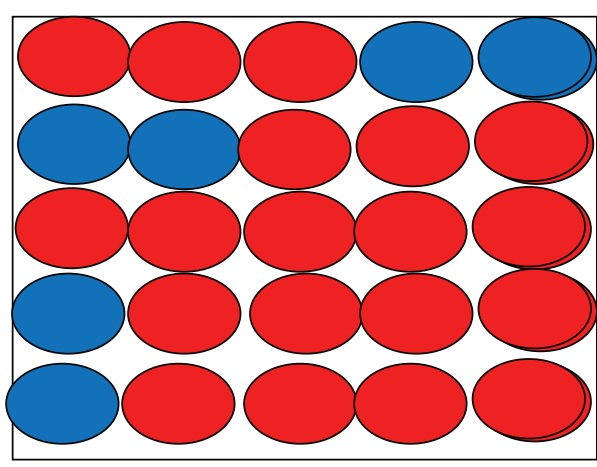

(a)

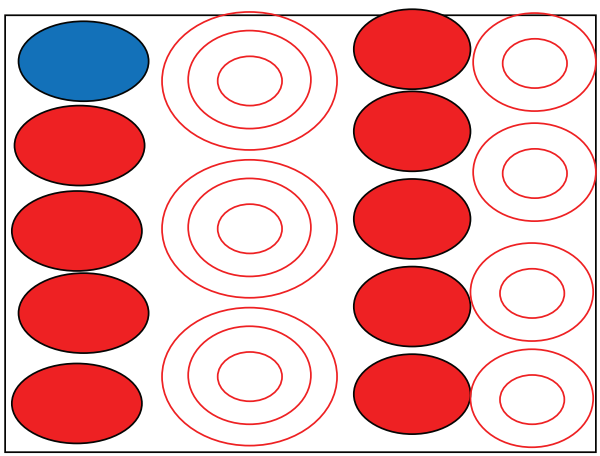

(c)

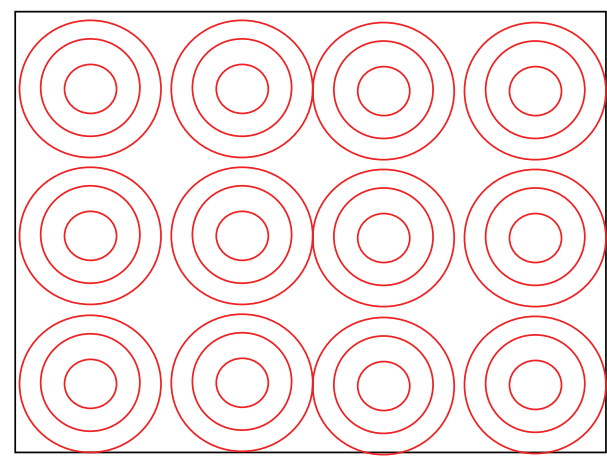

(b)

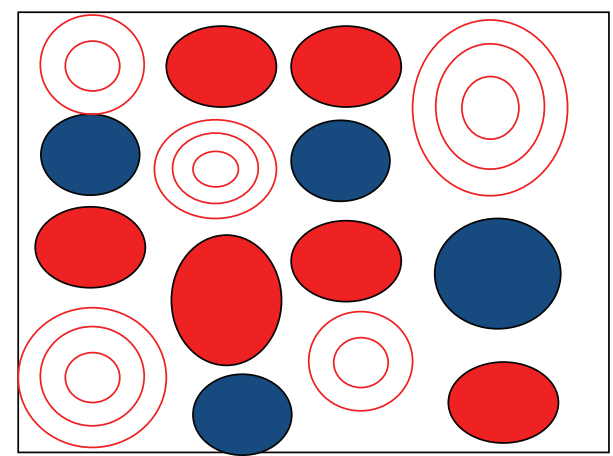

(d)

FIGURE 2: Different CNT interconnect geometries: (a) segregated SWCNT bundle, (b) segregated MWCNT bundle, (c) proportionately mixed CNT bundle, and (d) randomly mixed CNT bundle.

TABLE 1: Sizing ratio used for simulation study.

\begin{tabular}{lccccccccccc}
\hline Year & 2012 & 2013 & 2014 & 2015 & 2016 & 2017 & 2018 & 2019 & 2020 & 2021 & 2022 \\
\hline Width $(\mathrm{nm})$ & 36 & 32 & 28 & 25 & 22 & 20 & 18 & 16 & 14 & 13 & 11 \\
Delay of the copper (ps/um) & 0.85 & 1.13 & 1.56 & 1.79 & 2.48 & 3.12 & 3.66 & 4.98 & 7.11 & 7.27 & 11.44 \\
\hline
\end{tabular}

sisting of segregated SWCNTs, unsegregated SWCNTs of random diameters, segregated MWCNTs, and randomly mixed SWCNTs and MWCNTs as per ITRS predicted dimensions which are discussed in Table 1. The term segregated means bundles of CNTs with identical diameters while unsegregated means bundles of CNTs with different diameters. The performances of all these CNT geometries is evaluated using the tool carbon nanotube interconnect analyzer (CNIA) [11].

Section 4.1 discusses the calculation of various simulation parameters used for the creation of database which contains the performance of highly segregated bundles. Using this data, further analysis was carried out for revealing effect of mixing CNTs on interconnects performance. The results obtained are discussed in Section 4.2.

4.1. Segregated versus Unsegregated Random Mixed Bundles. The performance of 12 different CNT bundled segregated and unsegregated geometries was evaluated. The following geometries of segregated and unsegregated structures for metallic tube ratios of 1 and 0.4 (fully and partially metallic
CNTs) were taken into consideration for evaluation of performance:

(i) SWCNTs with tube diameters of $1.5 \mathrm{~nm}$,

(ii) Bundles made of unsegregated SWCNTs diameters varying randomly with a standard deviation of $1.25 \mathrm{~nm}$ and average diameter of $1.5 \mathrm{~nm}$,

(iii) bundles made of segregated MWCNTs of constant $D_{\min } / D_{\max }$ ratio (ratio of inner tube diameter to outer tube diameter of MWCNT) which were evaluated for $D_{\min } / D_{\max }$ ratios of 0.35 and 0.85 ,

(iv) randomly mixed, unsegregated bundles of SWCNTs and MWCNTs bundles made with tube diameters varying randomly with a standard deviation of $1.25 \mathrm{~nm}$ and average diameter of $1.5 \mathrm{~nm}$ for $D_{\min } / D_{\max }$ ratio as 0.35 and 0.85 .

The performance variations of these interconnect geometries from both local (i.e., $10 \mathrm{~nm}$ ) and global level (i.e., $10 \mu \mathrm{m}$ ) were evaluated. These studies revealed that fully metallic, random mixed CNT bundles made of minimum $D_{\min } / D_{\max }$ ratio 


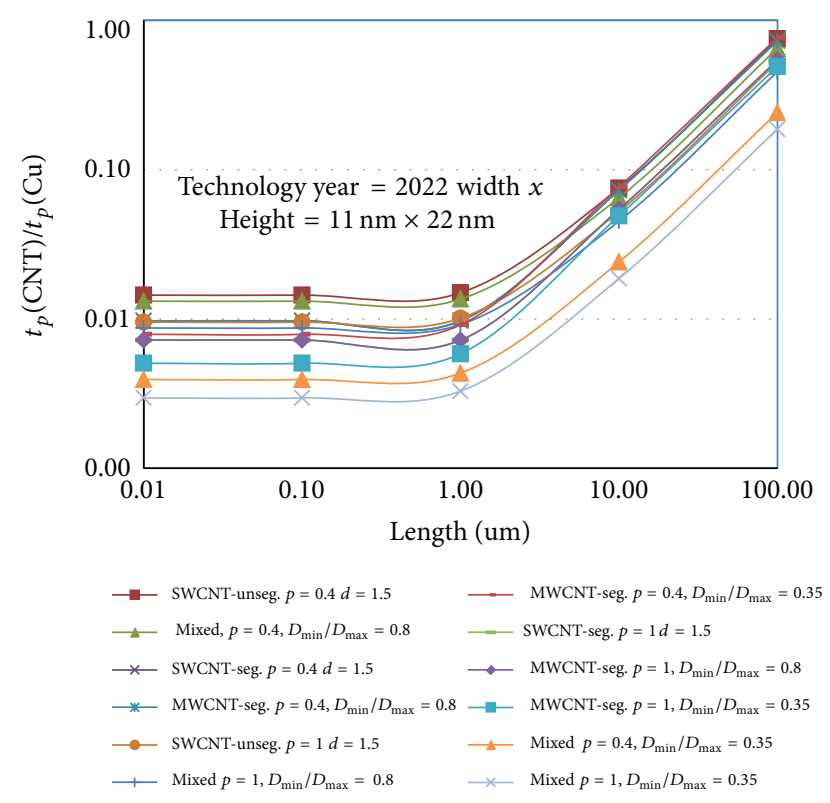

FIGURE 3: Ratio of propagation delay of CNT bundles with copper.

yield the best performance at all levels as shown in Figure 3. This is due to the availability of the maximum number of channels. The legends indicated in Figure 3 are marked in their descending order of performance as offered by various geometries. The worst case propagation delay was shown by bundles of unsegregated partially metallic SWCNTs. At both local and global levels, interconnects made of mixed CNTs performed better than other counterparts by an order of one. This improvement factor increased for densely packed bundles. In order to verify the applicability of these interconnects geometries against copper in futuristic technology nodes, the delays were compared with that of copper of the same dimension. Since copper shows its worst performance at the lowest technological node, we have considered the case of interconnects geometries for the prediction of year 2022.

It was found that even the CNT bundles with the worst performance were better than copper for the entire range of interconnect length improvements by an order two to three for interconnect length equal to or less than the mean free path length of CNT (i.e., $1 \mu \mathrm{m}$ ), with all CNT interconnect geometries.

4.2. Performance of Proportionately Mixed CNT Bundle. The performance of segregated bundles made of SWCNTs of constant diameters and MWCNTs of constant diameter ratio was evaluated. This data was used as the basic database for evaluating the performance of proportionately mixed bundles. Twelve different sets of values were obtained for interconnect geometries made using segregated SWCNT bundles containing CNTs of constant tube diameter for metallic tube ratios of $0.4,0.7$ and 1 . The various diameters of the CNTs considered for the creation of database were $1 \mathrm{~nm}, 3 \mathrm{~nm}, 5 \mathrm{~nm}$, and $7 \mathrm{~nm}$. Similarly, bundled segregated MWCNTs metallic MWCNTs of varying $D_{\min } / D_{\max }$ ratio and average diameter of the tubes in a bundle were considered. The values of outer diameter $D_{\max }$ used for the creation of database for MWCNTs were to $3 \mathrm{~nm}, 5 \mathrm{~nm}$, and $7 \mathrm{~nm}$ cases. In each case we also considered a variation in $D_{\min }$ (minimum diameter of the inner tube). In each case we considered the worst case condition of MWCNTs with only two concentric cylinders spaced apart by van der Waals distance and we considered that MWCNTs are made with multiple concentric cylinders spaced apart by van der Waals distance with a $D_{\min }$ close to $1 \mathrm{~nm}$ such that that each MWCNT has an inner tube with a diameter close to $1 \mathrm{~nm}$ for the best case analysis. The number of concentric cylinders in such case is given in (11).

The number of tubes in a MWCNT is denoted as $N_{\text {shell }}$ and is

$$
N_{\text {shell }}=1+\frac{D_{\max }-D_{\min }}{2 \delta} .
$$

$D_{\max }-D_{\min }$ are minimum and maximum diameters of the tubes in a MWCNT and $\delta$-van der Waals distance.

The number of CNTs in a densely packed bundle $N_{\text {bundle }}$ is calculated as per the studies given by Srivastava and Banerjee [4]. Simulation parameters such as tube density and average diameter were calculated for each case using (12) and (13), respectively:

$$
\text { Tube Density }=\frac{N_{\text {bundle }}}{\text { Area of the bundle }} .
$$

In case segregated SWCNTs average diameter is considered the diameter of the CNT, in case of MWCNTs it is calculated as

$$
D_{\text {Avg }}=\frac{\sum D_{\text {Total }}}{N_{\text {shell }}}
$$

where

$$
\begin{aligned}
\sum D_{\text {Total }}= & D_{(\text {Min })}+D_{(\operatorname{Min}+2 \delta)}+D_{(\operatorname{Min}+4 \delta)} \\
& +\cdots+D_{(\operatorname{Min}+2 n \delta)} .
\end{aligned}
$$

Hence we created six different sets of values for the performance of CNT bundles made using segregated SWCNTs/MWCNTs consisting of unique nature. The performance matrix and parasitic effects of these bundled structures were extracted with the help of carbon nanotube interconnect analyzer (CNIA) based on the input parameters for each case as discussed above. This database was used for evaluating performance of proportionally mixed CNT bundles.

The studies were carried out on three types of mixed CNT bundles (i.e., a mix of SWCNTs of different diameter, a mix of MWCNTs of varying $D_{\min } / D_{\max }$ ratio, or a mix of SWCNTs and MWCNTs).

In order to study the variations in performance due to mixing, the performance of segregated bundles made of similar kinds of SWCNTs or MWCNTs was used as the basic database. The behavioral variations of bundled CNTs on proportionate mixing were evaluated by

$$
\rho_{\text {mixed }}=\left(r_{1} \rho_{1}+r_{2} \rho_{2}+r_{3} \rho_{3}+\cdots+r_{N} \rho_{N}\right),
$$

where $\rho_{\text {mixed }}=$ delay of proportionately mixed bundle formed by mixing $N$-kinds of segregated CNTS and $r_{N}=$ ratio of the $N$ th sample used for mixing and where

$$
r_{1}+r_{2}+r_{3}+\cdots+r_{N}=1
$$


and $N=$ number of segregated combinations considering mixing and $\rho_{N}$-Propagation delay of each segregated structures with the same $W * H$ bundle dimension.

The performance of four different types of proportionate mixed CNTs is evaluated as below.

Case 1. A bundle which is formed by a mix of only segregated metallic SWCNTs of various diameters with combinations of tubes with lowest, middle, and highest diameter cases (i.e., $1 \mathrm{~nm}, 3 \mathrm{~nm}$, and $7 \mathrm{~nm}$ ).

Case 2. A bundle which is formed by a mix of only segregated metallic MWCNTs with various $D_{\min } / D_{\max }$ (lowest ratio, middle ratio, and maximum ratio) ratios.

Case 3. A mixed bundle comprised of two lowest diameters metallic SWCNTs and two MWCNTs with lowest $D_{\min } / D_{\max }$ ratio in equal proportion (for evaluating the best case performance of mixing CNTs).

Case 4. A combination of metallic SWCNTs with MWCNTs, a mix of two sets of largest diameters metallic SWCNTs with another two MWCNTs with largest $D_{\min } / D_{\max }$ in equal proportion (for evaluating worst case performance).

This selection of mixing indicated the relative performance of the bundles made only with a mix of SWCNTs or MWCNTs, enabling us to compare their performance with best and worst case performance of mixed bundle formed by mixing SWCNTs and MWCNTs. Effectively these data yield information about the range in which performance of these bundles can vary. The comparative performances of these mixed bundles are discussed below.

4.2.1. Proportionately Mixed versus Segregated Bundles. The propagation delay of various types of mixed CNT bundles as discussed above was calculated based on the expression given in (15). These were compared with best and worst case available performance of segregated CNTs. The delay offered for various interconnect lengths in all the cases discussed above is shown in Figures 4 and 5. The minimum propagation delay of mixed bundles is observed in Case 3, where two lowest diameters metallic SWCNTs and two MWCNTs with lowest $D_{\min } / D_{\max }$ ratio are mixed in equal proportion and the worst case performance of mixed CNTs was observed in Case 4 , where two sets of largest diameters metallic SWCNTs with another two MWCNTs with largest $D_{\min } / D_{\max }$ are mixed in equal proportion. These results also give an indication that the best performance is attained by bundles of lowest diameter, segregated fully metallic SWCNTs, and the worst performance corresponds to segregated SWCNT bundles of tubes with highest diameter and lowest metallic tube ratio. This occurs because the number of channels in the bundles is a direct function of diameter of the tubes. Figures 4 and 5 give a comparison of the propagation delay of the mixed bundles with the best and worst case performance of segregated CNT bundles at both local and semiglobal interconnect levels. Bundle size is fixed as per the interconnect dimensions predicted by ITRS and is given in Table 1 . The performance

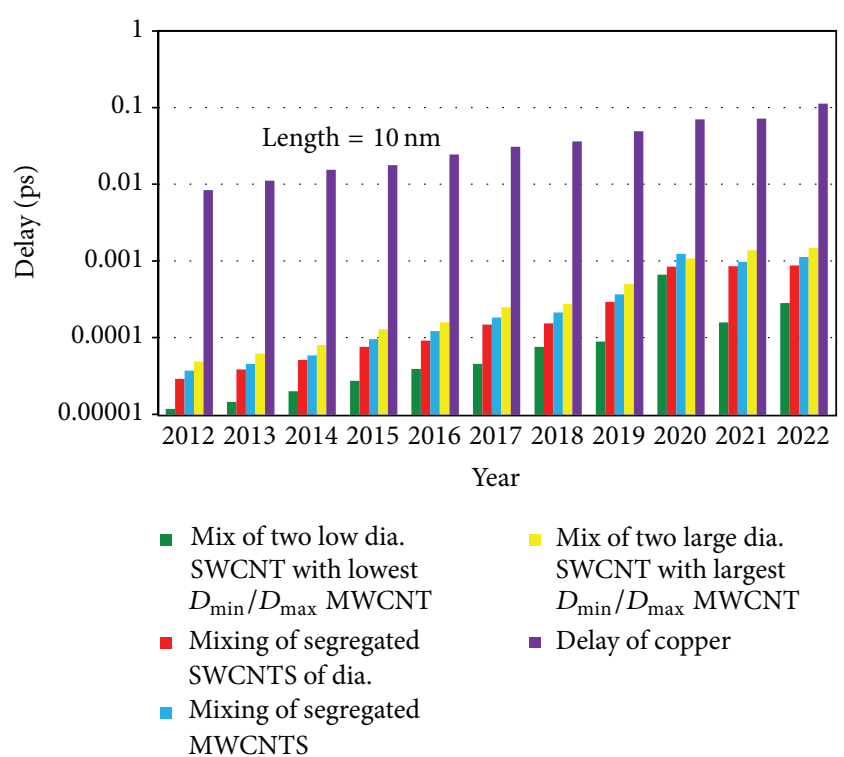

FIGURE 4: Propagation delay of mixed CNT bundle versus segregated bundle at local level.

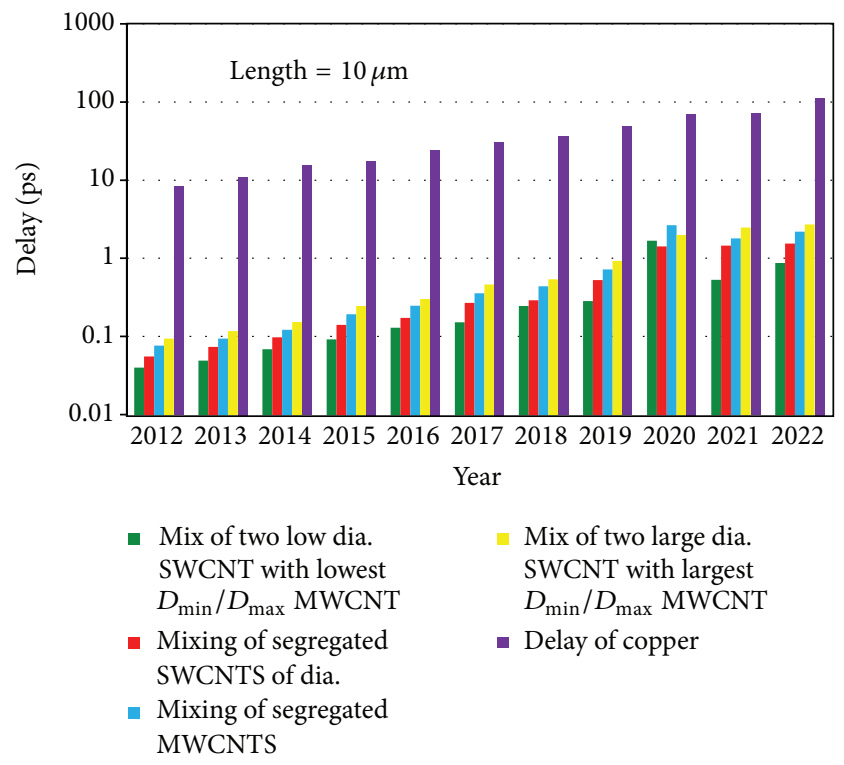

FIGURE 5: Propagation delay of mixed CNT bundle versus segregated bundle at semiglobal level.

of the mixed SWCNTs of different diameters shows only a variation of difference of order one from the best case performance offered by segregated bundles. At the same time the propagation delay is three orders less than that of copper.

The worst case performance of bundles which occurs when two sets of largest diameters metallic SWCNTs are mixed in equal proportion with two MWCNTs of maximum $D_{\min } / D_{\max }$ exhibited a variation of order two from the best case performance of segregated bundles. However this was two orders less than that of copper. Similarly it was found 


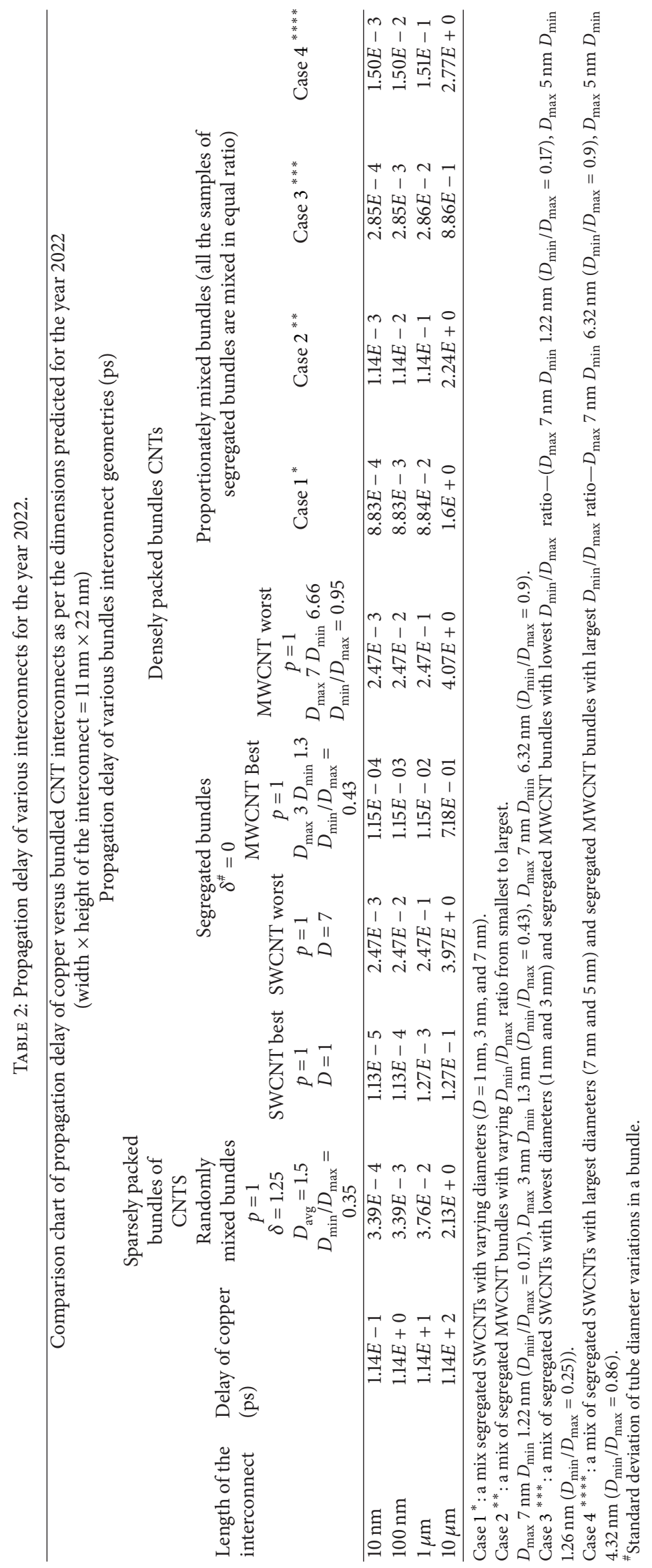


that all CNT geometries at global level also outperform copper. Mixed bundles of various MWCNTs have the same performance as the best case mix of SWCNTs and MWCNTs. The performance offered by various mixed configurations is almost equal. These results indicate that diameter controlled synthesis of CNTs is relatively more productive than segregation since this can reduce cost and time of production, while keeping the relative performance at the same level.

\section{Conclusion}

This work tried to comprehend the variation in performance and reliability of bundled CNTs as IC interconnects, as different types of CNTs were used for the construction of bundle structure. This work also aimed to answer the question whether segregation of CNT is absolutely necessary before the construction of interconnects or it can be readily used in mixed form, as it is synthesized. Consolidated variations of propagation delay of different interconnect geometries are given in Table 2. The basic analytical results of the performance of randomly mixed bundles which are sparsely packed were found to be better than that of segregated bundles. All geometries of CNT interconnect bundles with dimensions as per ITRS predictions till 2022 are found to perform better than copper except segregated SWCNT bundles constructed with tubes of higher diameters. The best performance was that of fully metallic segregated bundles of SWCNTs made with lowest tube diameter. The main observation made was that mixed bundles made of various combinations of CNTs had performance close to the best case performance observed from segregated SWCNTs. Moreover propagation delay of these densely packed mixed CNT bundles was found to be better than the delay of copper interconnects of the same dimensions by an order of two to three. This indicates that for extracting optimum performance vide cost matrix industry should focus more on diameter controlled synthesis method for the synthesis of CNTs rather than segregation.

\section{Conflict of Interests}

The authors declare that there is no conflict of interests regarding the publication of this paper.

\section{References}

[1] International Technology Roadmap for Semiconductors(ITRS), 2007, http://public.itrs.net.

[2] H. Li, C. Xu, N. Srivastava, and K. Banerjee, "Carbon nanomaterials for next-generation interconnects and passives: physics, status, and prospects," IEEE Transactions on Electron Devices, vol. 56, no. 9, pp. 1799-1821, 2009.

[3] A. Naeemi and J. D. Meindl, "Performance modeling for carbon nanotube interconnects," in Carbon Nanotube Electronics, A. Javey and J. Kong, Eds., chapter 7, pp. 163-190, Springer, 2009.

[4] N. Srivastava and K. Banerjee, "Performance analysis of carbon nanotube interconnects for VLSI applications," in Proceedings of the IEEE/ACM International Conference on Computer-Aided Design (ICCAD '05), pp. 383-390, November 2005.
[5] S. Sato, M. Niheri, A. Mimura et al., "Novel approach to fabricating carbon nanotube via interconnects using size-controlled catalyst nanoparticles," in Proceedings of the International Interconnect Technology Conference, pp. 230-232, June 2006.

[6] T. Inoue, I. Gunjishima, and A. Okamoto, "Synthesis of diameter-controlled carbon nanotubes using centrifugally classified nanoparticle catalysts," Carbon, vol. 45, no. 11, pp. 21642170, 2007.

[7] A. Naeemi and J. D. Meindl, "Compact physical models for multiwall carbon-nanotube interconnects," IEEE Electron Device Letters, vol. 27, no. 5, pp. 338-340, 2006.

[8] W. Wang, S. Haruehanroengra, L. Shang, and M. Liu, "Inductance of mixed carbon nanotube bundles," Micro \& Nano Letters, vol. 2, no. 2, pp. 35-39, 2007.

[9] S. Haruehanroengra and W. Wang, "Analyzing conductance of mixed carbon-nanotube bundles for interconnect applications," IEEE Electron Device Letters, vol. 28, no. 8, pp. 756-759, 2007.

[10] P. J. Burke, "Lüttinger liquid theory as a model of the gigahertz electrical properties of carbon nanotubes," IEEE Transactions on Nanotechnology, vol. 1, no. 3, pp. 129-144, 2002.

[11] S. Tanachutiwat and W. Wang, 2009, http://nanohub.org/ resources/cnia.

[12] M. K. Majumder, B. K. Kaushik, and S. K. Manhas, "A comparative study of SWNT bundle and MWNT in terms of area and propagation delay for global interconnects," International Journal of Contemporary Research in Engineering and Technology, vol. 1, no. 1, pp. 45-60, 2011.

[13] A. Giutiniani, "Modeling issues and performance analysis of high-speed interconnects based on a bundle of SWCNT," IEEE Transactions on Electron Devices, vol. 57, no. 8, pp. 1978-1985, 2010.

[14] F. Liang, G. Wang, and W. Ding, "Estimation of time delay and repeater insertion in multiwall carbon nanotube interconnects," IEEE Transactions on Electron Devices, vol. 58, no. 8, pp. 27122720, 2011. 

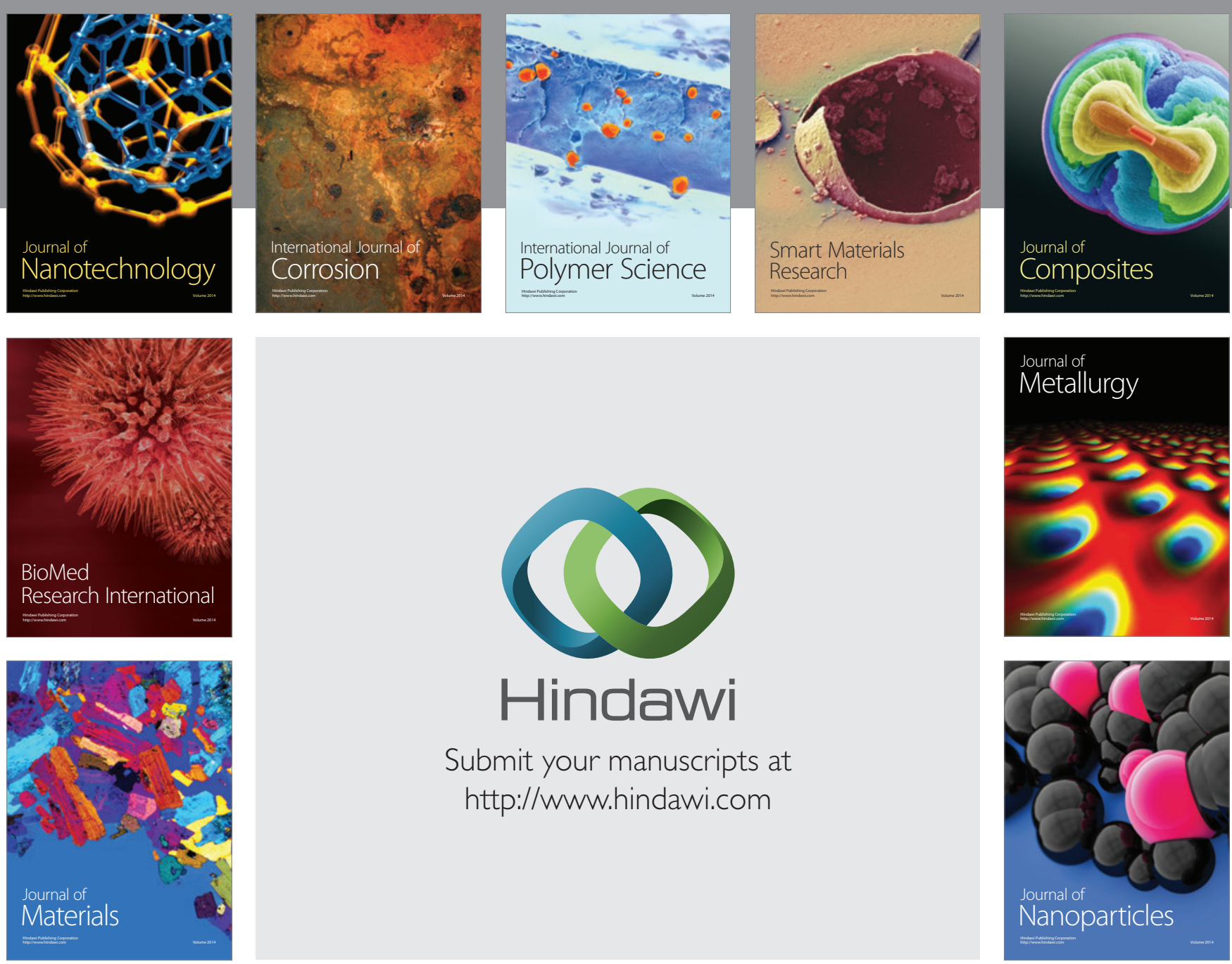

Submit your manuscripts at http://www.hindawi.com
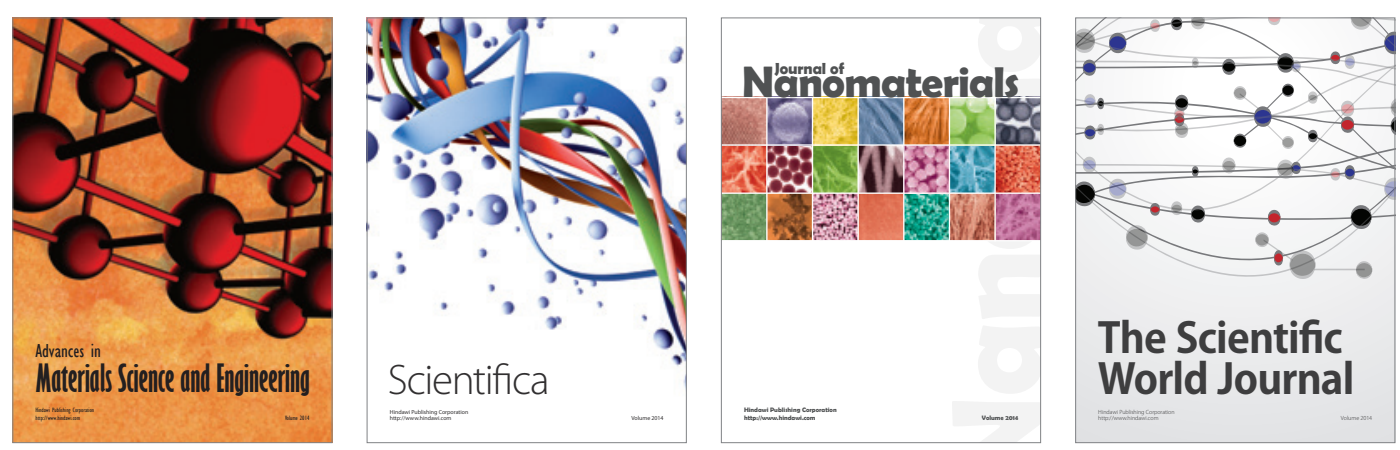

\section{The Scientific World Journal}
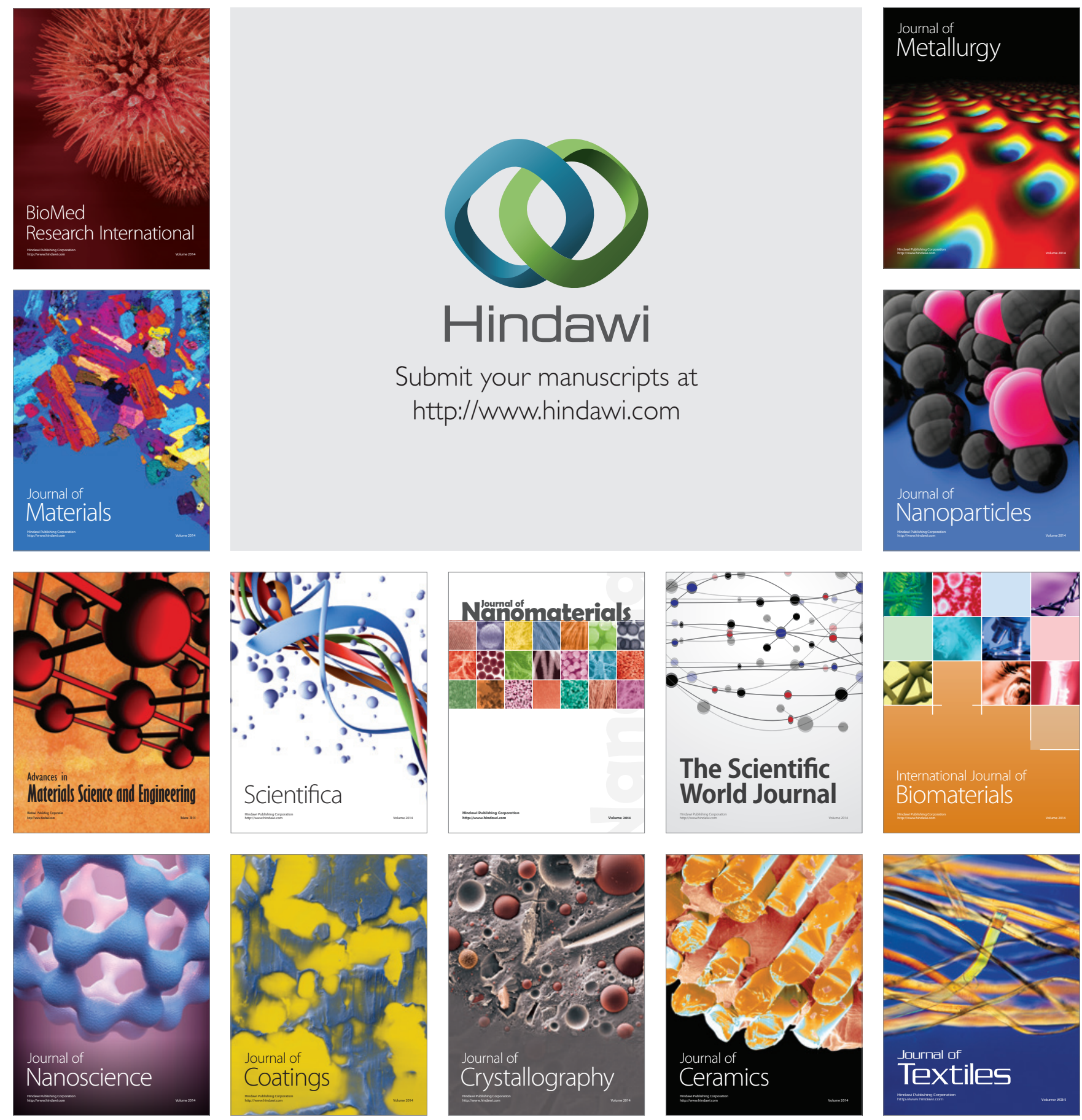